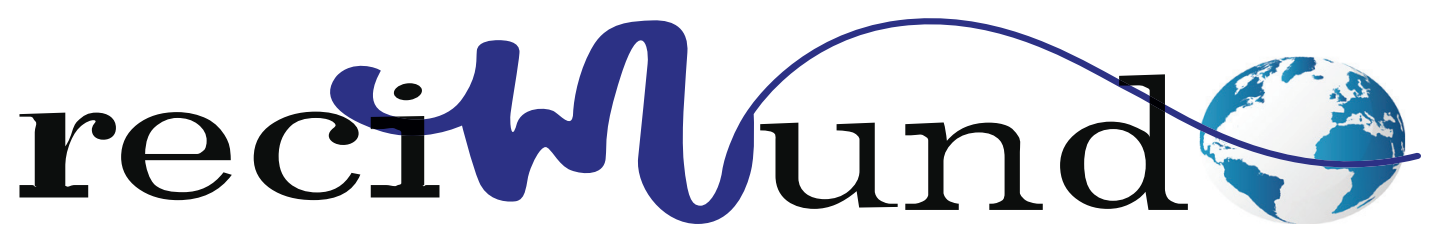

Revista Científica Mundo de la Investigación y el Conocimiento

DOI: 10.26820/recimundo/5.(2).julio.2021.278-292

URL: https://recimundo.com/index.php/es/article/view/1264

EDITORIAL: Saberes del Conocimiento

REVISTA: RECIMUNDO

ISSN: 2588-073X

TIPO DE INVESTIGACIÓN: Artículo de revisión

CóDIGO UNESCO: 32 Ciencias Médicas

PAGINAS: $278-292$

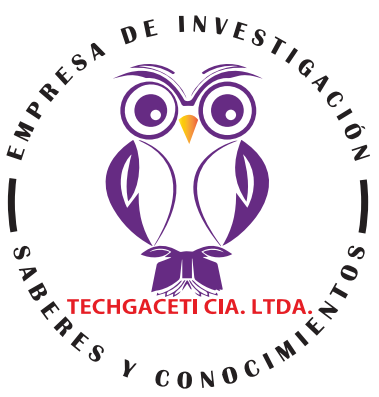

\title{
Monitorización invasiva y no invasiva en pacientes ingresados a $\mathrm{UCl}$
}

Invasive and non-invasive monitoring in patients admitted to the ICU Monitoramento invasivo e não invasivo em pacientes internados na UTI

Nelson Geovanny Moreno Sasig;; José Ricardo Vélez Muentes²; Manuel Antonio Campuzano Franco3; Jaime Roberto Zambrano Córdova4; Ronal Gabriel Vera Pinargote ${ }^{5}$

\section{RECIBIDO: 11/04/2021 ACEPTADO: 15/06/2021 PUBLICADO: 30/07/2021}

1. Médico General; Médico Residente de Terapia Intensiva; Hospital General IESS Santo Domingo; Ecuador; dr.moreno.nelson@gmail.com; (D) https://orcid.org/0000-0002-9240-8681

2. Médico General; Médico Residente de Terapia Intensiva; Hospital General IESS Santo Domingo; Ecuador; ricardovelezmuentes@gmail.com; iD https://orcid.org/0000-0002-5314-8506

3. Médico General; Médico Residente de Terapia Intensiva; Hospital General IESS Santo Domingo; Ecuador; manucam86@ hotmail.com; (iD https://orcid.org/0000-0003-4519-7639

4. Médico Cirujano; Médico Residente de Terapia Intensiva; Hospital General IESS Santo Domingo de los Tsachilas; Ecuador; zambrano.roberto@gmail.com; (iD) https://orcid.org/0000-0001-6609-0959

5. Médico General; Médico Residente de Terapia Intensiva; Hospital General less Santo Domingo; ronal_vera@hotmail.com; (iD https://orcid.org/0000-0001-7306-9728

\section{CORRESPONDENCIA}

Nelson Geovanny Moreno Sasig

dr.moreno.nelson@gmail.com

Santo Domingo; Ecuador 


\section{RESUMEN}

La monitorización del paciente crítico es fundamental para lograr optimizar la hemodinámica, la ventilación, la temperatura, la nutrición y el metabolismo del paciente. La mayoría de las monitorizaciones se realizan sobre procedimientos invasivos como cateterización venosa central y periférica, abordaje arterial, sondajes nasogástricos y vesicales, para obtener información hemodinámica, ventilatoria y metabólica. No obstante, también existen equipos tecnológicos especializados y nuevos algoritmos que pueden ayudar a obtener la mayor cantidad de variables y parámetros de manera no invasiva para lograr el mismo objetivo. El presente artículo no pretende establecer criterios comparativos sobre ventajas y desventajas de las técnicas de monitoreo invasivo y no invasivo, sino presentar, a modo descriptivo, la disponibilidad de las mismas como alternativas en el cuidado del paciente crítico. Para ello, se llevó a cabo una exhaustiva investigación en diferentes literaturas para presentar el contenido idóneo, con aportación importante al conocimiento del tema.

Palabras clave: Monitorización, Hemodinámica, Invasivo, UCI, Monitoreo.

\section{ABSTRACT}

Critical patient monitoring is essential to optimize the patient's hemodynamics, ventilation, temperature, nutrition, and metabolism. Most of the monitoring is performed on invasive procedures such as central and peripheral venous catheterization, arterial approach, nasogastric and bladder tubes, to obtain hemodynamic, ventilatory and metabolic information. However, there are also specialized technological equipment and new algorithms that can help obtain the greatest number of variables and parameters in a non-invasive way to achieve the same objective. This article does not intend to establish comparative criteria on the advantages and disadvantages of invasive and non-invasive monitoring techniques, but rather to present, in a descriptive way, their availability as alternatives in the care of the critical patient. For this, an exhaustive investigation was carried out in different literatures to present the ideal content, with an important contribution to the knowledge of the subject.

Keywords: Monitoring, Hemodynamics, Invasive, ICU, Monitoring.

\section{RESUMO}

O monitoramento de pacientes críticos é essencial para otimizar a hemodinâmica, ventilação, temperatura, nutrição e metabolismo do paciente. A maior parte da monitoração é realizada em procedimentos invasivos como cateterismo venoso central e periférico, abordagem arterial, sonda nasogástrica e vesical, para obtenção de informações hemodinâmicas, ventilatórias e metabólicas. No entanto, também existem equipamentos tecnológicos especializados e novos algoritmos que podem ajudar a obter o maior número de variáveis e parâmetros de forma não invasiva para atingir o mesmo objetivo. Este artigo não pretende estabelecer critérios comparativos sobre as vantagens e desvantagens das técnicas de monitoramento invasivo e não invasivo, mas sim apresentar, de forma descritiva, sua disponibilidade como alternativa no cuidado ao paciente crítico. Para isso, foi realizada uma investigação exaustiva em diferentes literaturas para apresentar o conteúdo ideal, com uma importante contribuição para o conhecimento do assunto.

Palavras-chave: Monitoramento, Hemodinâmica, Invasiva, UTI, Monitoramento. 


\section{Introducción}

Dentro de las Unidades de Cuidados Intensivos (UCI), la monitorización del paciente crítico es fundamental para el cuidado idóneo en estos casos para optimizar la hemodinámica, la ventilación, la temperatura, la nutrición y el metabolismo del paciente, ya que es la clave para mejorar la supervivencia del mismo. De hecho, el criterio médico toma como valoración decisiva el suministro de oxígeno a los tejidos de acuerdo con sus necesidades metabólicas para alimentar la respiración mitocondrial y, y de esta manera, mantener la vida (Weiner et al, 2021).

En tal sentido, tanto la oxigenación como la perfusión deben ser monitoreadas sea cual sea en la estrategia de reanimación implementada. $Y$ el concepto que ha emergido al respecto como monitorización hemodinámica, tiene como finalidad mejorar la macrocirculación a través de la optimización secuencial de la función cardíaca para que, idóneamente, luego se valorare la perfusión/oxigenación en parámetros específicos, en cualquier diseño estratégico escogido por los especialistas del área (Almela et al, 2015).

Por otro lado, es menester el mantenimiento de la temperatura normal y su control periódico es fundamental, así como la monitorización respiratoria de los pacientes de UCI ventilados, la cual debe incluir evaluación seriada del intercambio de gases, de la mecánica del sistema respiratorio, valoración continua para la preparación del paciente para su liberación de la ventilación invasiva con presión positiva (Warttig et al 2018).

Sumado a todo lo anterior, está el seguimiento de los cuidados nutricionales y metabólicos para controlar la entrega de nutrientes, la adecuación entre las necesidades y la entrega de energía y la glucosa en sangre (De Waele et al , 2018).

Actualmente, la mayoría de las UCI del mundo disponen de diferentes técnicas de mo- nitoreo del paciente crítico realmente efectivas para llevar a cabo un control sistemático de variables fisiológicas, que se miden con el objeto de detectar, reconocer y corregir tempranamente alteraciones de aparatos sistemas que podrían provocar posibles complicaciones (Weiner et al, 2021).

Dichas técnicas de monitoreo suelen ser invasivas, ya que la mayoría se realizan sobre procedimientos invasivos (cateterización venosa central y periférica, abordaje arterial, sondajes nasogástricos y vesicales, etc). No obstante, también se cuenta con técnicas de monitoreo no invasivos que logran obtener parámetros fundamentales, ya que hoy día se cuenta con equipos tecnológicos especializados y nuevos algoritmos, que efectivamente ayuden al intensivista a monitorear eficientemente al paciente (Warttig et al, 2018).

Por lo tanto, el presente artículo describe las principales técnicas de monitoreo invasivos y no invasivos, que permitan la valoración adecuada de la perfusión/ oxigenación y de los controles de temperatura, respiratorios, nutricionales y metabólicos, a partir de un análisis de contenido de diferentes literaturas, con la finalidad de crear y extender conocimiento del tema investigado.

\section{Metodología}

El presente artículo fue realizado a partir de un análisis descriptivo de las diferentes literaturas consultadas a través de bases referenciales como Google Académico, The Lancet, Cochrane Library, y de bibliografías puntuales, de fecha superior al 2015, recolectando la información sobre las diferentes técnicas de monitoreo de pacientes en cuidados intensivos a partir de criterios de selección que abarcara el monitoreo invasivo y no invasivo de pacientes críticos.

Desde un enfoque descriptivo y documental se desarrolló el contenido del presente artículo para presentan las diferentes téc- 
nicas invasivas y no invasivas utilizadas en la realidad actual, sin realizar comparativas de efectividad relevantes entre las mismas, sino más bien se caracterizaron las diferentes monitorizaciones disponibles para pacientes críticos, desde un punto referencial y utilitario.

\section{Resultados}

\section{El monitoreo de paciente crítico}

Cuando un paciente es ingresado en la $\mathrm{UCl}$, los profesionales dotados de conocimiento y habilidad se abalanzan sobre él con arsenales de artefactos tecnológicos para monitorizarlo, diagnosticarlo, y tratarlo, y la mayoría de estas prácticas resultan ser invasivas. El riesgo tomado obedece a una normativa legal, que el profesional intensivista debe conocer y que el paciente y familiares deben consentir, ya que la intención es salvar la vida, por eso, generalmente el riesgo se toma sin vacilar (Aguilar \& Martinez, 2017).

La ciencia médica actual conjuntamente con los adelantos tecnológicos han desarrollado técnicas de monitorización del paciente crítico, para llevar a cabo de manera objetiva la atención intensiva que este requiere, ya que se encuentran en el nivel muy alto de agudeza, con tendencia a eventos clínicos o complicaciones potenciales durante el curso de su estancia en la UCI (Almela et al, 2015).

Este monitoreo del paciente crítico es un control sistemático de variables fisiológicas que se miden con el objeto de detectar, reconocer y corregir tempranamente alteraciones de aparatos sistemas que podrían provocar posibles complicaciones. De acuerdo con Weiner et al (2021), en general, el monitoreo en terapia intensiva busca mantener y restaurar la función de órganos vitales, y consiste en:

\section{Monitoreo Respiratorio:}

Se realiza en aquellos pacientes de $\mathrm{UCI}$ ventilados, el cual consiste en una evaluación seriada del intercambio gaseoso, de la mecánica del sistema respiratorio, con el fin de prepararlos para la liberación de la ventilación invasiva con presión positiva (Weiner et al, 2021).

En su sentido más amplio, esta monitorización a lo largo del tiempo puede ayudar a minimizar las complicaciones asociadas al ventilador, optimizar la sincronía entre el ventilador y el paciente; también proporciona pistas importantes sobre las posibles causas de sonido de la alarma así como de los cambios en las condiciones de los pacientes (Weiner et al, 2021).

Por lo tanto, un requisito previo para este enfoque es una buena comprensión de la fisiología detrás de las variables que se monitorean: Parámetros respiratorios (FiO2, frecuencia, espirometría, humedad y temperatura, espacio muerto, permeabilidad, presión, relación I/E, sensibilidad, alarmas), intercambio gaseoso, mecánica pulmonar, sincronía paciente-ventilador. La medición de dichos parámetros debe realizarse al inicio de la ventilación mecánica y posteriormente, a intervalos regulares, particularmente en pacientes que son difíciles de ventilar y oxigenar y que requieren sedación intensa y parálisis (Weiner et al, 2021).

\section{Monitoreo Hemodinámico:}

El monitoreo hemodinámico es la base fundamental de la atención para el paciente hemodinámicamente inestable, requiere de un multienfoque y su uso es tanto para la entidad específica como para su contexto. Tiene como objetivo proporcionar datos que permitana optimizar la oxigenación de los tejidos de los órganos terminales, y detener de forma eficaz la hipoxia tisular global, el shock y la insuficiencia multiorgánica (Weiner et al, 2021). 
Entre las variables estudiadas en la monitorización hemodinámica se encuentran la frecuencia cardíaca (FC), la presión venosa central (PVC), la presión arterial media (PAM), la presión arterial diastólica (PD), y la presión sanguínea sistólica (PS), y el gasto cardíaco (GC), Este último se considera fundamental para poder valorar la función cardíaca y monitorear la oxigenación de los tejidos, pudiendo así alertar sobre la hipoxia como posibilidad, como de otros aspectos (Weiner et al, 2021).

\section{Monitoreo Metabólico:}

Este seguimiento es de gran relevancia, ya que abundan las evidencias que señalan que un control glucémico deteriorado más una cobertura insuficiente de las necesidades proteicas y energéticas, están relacionados con fracasos clínicos en la UCI (Singer et al, 2019).

Según De Waele et al (2018), el monitoreo de la atención nutricional y metabólica en la $\mathrm{UCI}$ tiene tres objetivos principales:

a) Control de la entrega de macronutrientes (glucosa, proteínas, grasas) y micronutrientes (vitaminas y oligoelementos),

b) Evaluación de la adecuación entre la entrega y las necesidades energéticas.

c) Control glucémico.

Por lo tanto, al darse una adaptación temprana y estricta de la entrega de proteínas y energía a los objetivos nutricionales y una optimización del control glucémico, el monitoreo de los parametros como gasto energético total (GET), gasto energético basal (GEB), gasto energético en reposo (GER), consumo de oxígeno (VO2), producción de dióxido de carbono (VCO2), y por supuesto la medición de glicemia en sangre, podría mejorar la adherencia a las guías clínicas y el resultado clínico de los pacientes de la UCl (Singer et al , 2019).

\section{Monitoreo Neurológico:}

Se realiza en aquellos los pacientes críti- cos con lesión cerebral traumática que requieran un diagnóstico preciso y rápido de eventos patológicos intracraneales ( $\mathrm{Ci}$ terio et al, 2015).

De acuerdo con Carrillo y Garrido (2015), el objetivo del monitoreo neurológico multimodal consiste en la realización de undiagnóstico integral que permita ofrecer un tratamiento, a través del análisis de diferentes variables como la clínica, evaluación de la presión intracraneal, presión de perfusión cerebral, oximetría cerebral y savyo2 y el monitoreo electrofisiológico.

Dicho monitoreo neurológico podría mejorar el pronóstico del paciente ante una lesión cerebral traumática y aunque la implementación del monitoreo univariable no ha demostrado incremento en la sobrevida de los pacientes, el análisis multivariable se relaciona con un terapéutica más juiciosa y objetiva que ha permitido identificar tempranamente del tratamiento del paciente crítico (Carrillo E \& Garrido A, 2015).

\section{Monitoreo de Temperatura:}

Es importante el mantenimiento de la temperatura corporal normal en el paciente crítico, por lo que es menester controlarse periódicamente. De hecho, ensayos como el de Sessler (2008), han demostrado que incluso la hipotermia leve causa numerosos resultados adversos, como miocárdicos mórbidos consecuentes a la activación del sistema nervioso simpático. Asì mismo, coagulopatía, la infección de la herida quirúrgica, retraso en la cicatrización de la herida, retraso en la recuperación postanestésica, escalofríos, malestar del paciente y prolongación de su estadía en UCI ( Kipnis et al, 2015).

Por otro lado, se sabe también que los anestésicos generales producen una profunda reducción de la temperatura central como dosis dependiente, secundaria al deterioro de los mecanismos termorreguladores normales, a causa de la redistribución del calor 
corporal del núcleo a la periferia (Kipnis et al, 2015).

\section{Monitoreo de diuresis y balance hídrico:}

Es una monitorización importante que no solo aporta datos relevantes al monitoreo hemodinámico, sino que por ejemplo, la diuresis mesurable permite estar atentos a posibles infecciones nosocomiales, que podrían estar asociada a la cateterización vesical del paciente (Crashing Patient, 2020).

\section{Monitoreo sanguíneo:}

Es un monitoreo claramente invasivo que puede producir daño a las venas, y ocasionar dolor y anemia, pero permite detectar tempranamente cualquier alteración. Se realiza diariamente en toda unidad de cuidados intensivos, y generlamente, se realizan análisis de electrolitos y hemograma completo, concentraciones de magnesio, fosfato y calcio, así como hemocultivos en caso de fiebre. Si el paciente recibe nutrición parenteral total, se realiza estudio de enzimas hepáticas y perfil de coagulación semanal (Maqueda \& Pérez, 2018).

La mayoría de las monitorizaciones anteriormente descritas se realizan sobre procedimientos invasivos (cateterización venosa central y periférica, abordaje arterial, sondajes nasogastricos y vesicales, etc). Sin embargo, la monitorización sobre estos procedimientos, no necsariamente debe ser invasiva, ya que hoy día se cuenta con equipos tecnológicos especializados y nuevos algoritmos que pueden ayudar a obtener la mayor cantidad de variables y parámetros, que efectivamente ayuden al intensivista a monitorear eficientemente al paciente (Warttig et al, 2018).

A continuación, se presentan las diferentes técnicas invasivas y no invasivas para los principales monitoreos descrito, no a modo de determinar la efectividad de las mismas en el ámbito de las $\mathrm{UCl}$, porque, además, ello dependerá de la patología del paciente, de los recurso tecnológicos y del personal especializado con los que cuenta cada unidad, sino más bien para caracterizar las diferentes monitorizaciones disponbles para pacientes críticos actualmente.

\section{Monitorización invasiva y no invasiva}

Se habla de monitorización invasiva cuando los parámetros requeridos para la valoración del estado del paciente, implican la insición del tejido y abordar sobre el organismo para obtener la información necesaria para mantener vigilado y estable al paciente. Este tipo de monitoreo es parte de una sumatoria de riesgo de mortalidad para el paciente crítico que ya de por sí lidia con su delicado estado multidisfuncional sistémico, sin embargo, en muchos casos también supone la mejor manera de poderle estabilizar y darle oportunidad de vida (Naumann et al, 2016).

Por otro lado, la monitorización no invasiva se refiere a el control de parámetros obtenidos por medio de técnicas mecánica como la tensión arterial (TA), a través de un esfigmomanómetro, o la temperatura, a través del termemómetro, o también pueden ser obtenidas (y es lo más frecuente para el cuidado intensivo) a través de los monitores de signos vitales para UCI. De hecho, en la actualidad se dispone de estos equipos con configurados para medir y mostrar en pantalla más de 20 tipos de parámetros diferentes, provenientes de sensores y dispositivos que se colocan en el paciente (CareTaker, 2020).

\section{Monitoreo Respiratorio No Invasivo}

\section{Examen Clínico a través de la observa- ción:}

En la evaluación del paciente en ventilación mecánica es fundamental buscar y reconocer los signos clínicos a través del examen clínico, que incluye el monitoreo del tubo orotraqueal. Este examen debe ser exhaustivo y rápido para evidenciar o descartar cianosis; el patrón respiratorio como cla-

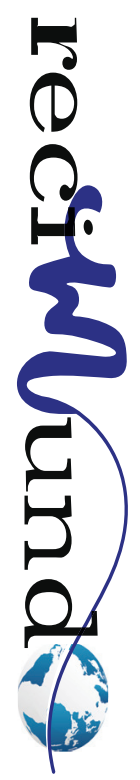


ve, ya que indica la sincronía del paciente y el esfuerzo realizado por el mismo; tos; agitación; la taquipnea puesto que es un fenómeno generalmente compensatorio de la hipoxemia; sudoración; uso de músculos accesorios; hipotensión de múltiples causas; aleteo nasal; tirajes universales; incremento de movimiento de extremidades, y respiración toracoabdominal paradójica (Weiner et al, 2021).

\section{Oximetría de pulso y Capnografía}

Un diagrama interesante propuesto por Jones y Jones (2020), ha permitido la interpretación de la conocida curva de disociación de oxígeno de la oxihemoglobina, en el monitoreo de las anormalidades del intercambio gaseoso con oximetría de pulso, el cual consiste en el uso SpO2/FiO2 utilizando $\mathrm{SpO} 2$ en lugar de $\mathrm{SaO} 2$ y reemplazando $\mathrm{PaO} 2$ por $\mathrm{FiO} 2$ para interpretar el cortocircuito de ventilación/perfusión (V/Q). De esta manera, sí un paciente ventilado mecánicamente con SpO2 a cierto porcentaje y a una FiO2 constante ya sea baja o alta y no hay cambios en la saturación al realizar maniobras ventilatorias para modificar el cortocircuito, se deben realizar otro tipo de maniobras ventilatorias. Este mecanismo proporciona una aproximación simple del cortocircuito, que no requiere ni muestras de sangre arterial ni un catéter de arteria pulmonar, sino un esquema simple, dinámico, con un monitoreo no invasivo del estado del intercambio gaseoso dada por lecturas individuales $\mathrm{SpO} 2, \mathrm{SaO} 2$ o PaO2 obtenidas a FiO2 constante. (Ortiz \& Dueñas, 2019).

En cuanto a la medición del CO2 espirado, el cual se mide a través de un aparato que se conecta entre el tubo del paciente y el ventilador mecánico, y tiene un cable que se conecta al monitor. La capnometría es el registro numérico del $\mathrm{CO} 2$ espirado, mientras que la capnografía presenta gráficamente, en forma de curva, la concentración de $\mathrm{CO} 2$ al final del gas exhalado de cada respiración. Este valor se utiliza para saber sobre la ventilación de los alveolos, y sus valores normales de la presión arterial de $\mathrm{CO} 2$ (PaCO2) son de entre 35 - 45 mmHg (Cediel et al, 2020).

Los avances en oximetría de pulso y en capnografía hacen cada vez menos necesario el uso de gases arteriales. Estas técnicas junto la saturación venosa continua, han permitido reducir la frecuencia de toma de gases sanguíneos (Cediel et al, 2020).

\section{Estimaciones de la mecánica del sistema respiratorio}

La monitorización de variables de la mecánica ventilatoria como la presión pico, presión meseta, distensibilidad, la resistencia y el trabajo respiratorio ayudan al profesional intensivista en el manejo del paciente ventilado. Para ello, es necesario tener el conocimiento sobre la relación entre presión, flujo, volumen y la mecánica del sistema respiratorio, la cual puede abordarse utilizando la ecuación simplificada de movimiento que establece que la presión (P) necesarios para administrar un volumen corriente se pueden calcular de la siguiente manera:

$$
P=\left(\frac{V_{T}}{C_{\mathrm{RS}}}\right)+\left(\frac{R_{\mathrm{RS}} \times V_{T}}{\mathrm{Ti}}\right)+\text { total PEEP, }
$$

donde $\mathrm{Vt}=$ volumen de corriente, y Crs= distensibilidad y resistencia generales del sistema respiratorio (RS), respectivamente, $\mathrm{Ti}=$ tiempo inspiratorio $\mathrm{y}=$ flujo inspiratorio y PEEP = presión positiva al final de la espiración (Ortiz \& Dueñas, 2019).

Los ventiladores modernos muestran curvas de tiempo de flujo, volumen y presión en tiempo real (ver figura 1), lo que permite al profesional de $\mathrm{UCl}$, la revisión diaria de estas curvas esenciales para evaluar si la configuración del ventilador es segura y se adapta a las condiciones de los pacientes (Ortiz \& Dueñas, 2019). 


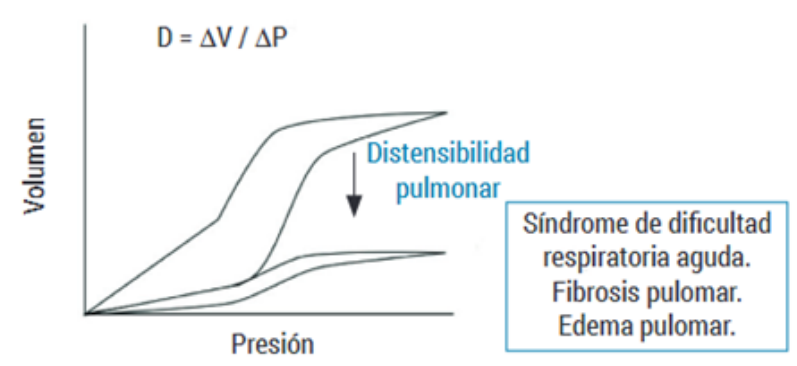

$\Delta \mathrm{V}$ : cambio de volumen; $\Delta \mathrm{P}$ : presión de conducción

Figura 1. Curva de ventiladores que muestra reduccion de la distensibilidad

Fuente: Ortiz \& Dueñas, (2019) (Ob. Cit).

El seguimiento de los cambios del sistema respiratorio a lo largo del tiempo puede ayudar a minimizar las complicaciones asociadas al ventilador, optimizar la sincronía entre el ventilador y el paciente y proporcionar pistas importantes sobre las posibles causas del sonido de la alarma y / o cambios en las condiciones de los pacientes.(Motta et al, 2017).

4. La tomografía computarizada (TC) y la ecografía torácica

La imagenología ha ido ganando importancia en el diagnóstico y seguimiento del paciente en ventilación mecánica. Dentro de este método entra la radiografía de tórax diaria, en la cual se evalúa la posición del tubo orotraqueal o cánula de traqueotomía, la presencia de neumotórax, controlar la posición de catéter de arteria pulmonar, del catéter central, también se busca evidencia de nuevos infiltrados, neumonía, SDRA, entre otros (Ortiz \& Dueñas, 2019).

También la ecografía pleural puede ser necesaria para evaluar la magnitud de los derrames y para escoger el mejor sitio para drenaje, de una manera más segura. Además, la ecografía pulmonar se ha propuesto como diagnóstico, monitorización y manejo de pacientes con neumonía y edema pulmonar.
Puede estar sumado a estas técnicas de monitoreo, la tomografía pulmonar, la cual se considera como un examen específico, en el que se puede evaluar la evolución de un SDRA, derrames pleurales, consolidación. Pero además, la tomografía por bioimpedancia eléctrica, con electrodos alrededor de la pared del tórax, sirve para evaluar las maniobras de reclutamiento (Ortiz \& Dueñas, 2019).

\section{Monitoreo Respiratorio Invasivo}

\section{Gasometría}

Hay que tener claro que el monitoreo hemodinámico va de la mano con el respiratorio, y otras monitorizaciones del paciente, ya que el organismo como tal es un sistema de órganos que se entremezclan y orquestan los unos a los otros. Así pues, no es obvio que una medición que se establezca como respiratoria, no sea parte de la hemodinámica, como es el caso de los gases en sangre.

Por ello, la técnica de cateterización o punción arterial y venosa para realizar la mesura de los gases arteriales o gasometría, sirve para el monitoreo de parámetros importantes. Según Ortiz \& Dueñas (2019), para el caso del paciente ventilado, los gases arteriales se deben medirse a diario, y cada vez que exista un cambio en la evolución del paciente, o cuando se modifiquen parámetros del ventilador.

De acuerdo con Cediel et al (2020), la gasometría evalúa de una manera estática:

a) La oxigenación de la sangre arterial con la presión parcial de oxígeno en la sangre arterial ( $\mathrm{PaO} 2)$.

b) La eficiencia de la membrana alveolocapilar con la diferencia alveoloarterial de oxígeno o con la relación arterioalveolar de oxígeno, o la relación entre la $\mathrm{PaO} 2$ y la fracción inspirada de oxígeno, reflejandose de manera sencilla y global la capacidad de difusión de la membrana, y que debe ser mayor de 300. 
MORENO SASIG, N. G., VÉLEZ MUENTES, J. R., CAMPUZANO FRANCO, M. A., ZAMBRANO CÓRDOVA, J. R., \& VERA PINARGOTE, R. G.

c) La producción de PCO2.

d) El estado ácido básico (Ver figura 2)

e) El contenido arterial de oxígeno en sangre, de una manera indirecta, mediante la fórmula:

$\mathrm{CaO} 2=(\mathrm{Hb} \times 1,39) \mathrm{SO} 2+(0,031 \times \mathrm{PaO} 2)$, donde $\mathrm{Hb}$ es la hemoglobina

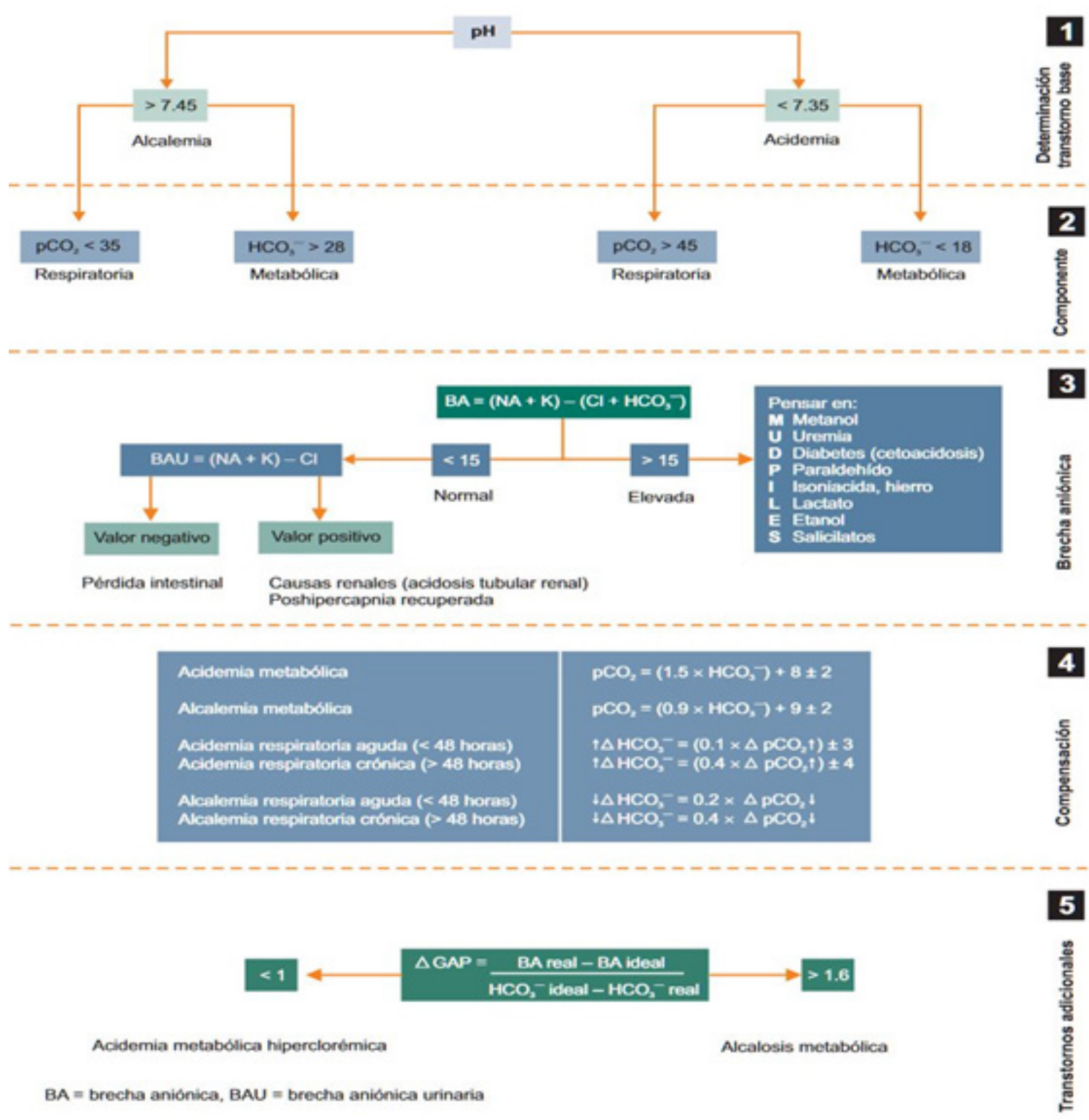

Figura 2. Interpretación de los trastornos ácido-base a partir de una Gasometría

Fuente: Márquez, et al (2012) (Ob Cit). 


\section{Monitoreo Hemodinámico No Invasivo}

\section{Bioimpedancia-biorreactancia electrica torácica}

La cardiografía de impedancia (ICG) o bioimpedancia está basada en la ley de Ohn, la cual determina cambios en el contenido líquido torácico apoyado en el diferencial de la conductividad/resistencia, a la propagación de un impulso eléctrico a través del tórax. De este modo, la variación del flujo sanguíneo en los grandes vasos produce las alteraciones en el voltaje y en la impedancia eléctrica torácica, de lo cual se traducen en los parámetros: gasto cardíaco (GC), volumen sistólico, resistencia vascular sistémica, parámetros de contractilidad ventricular y estándar de volemia (contenido de fluido torácico) (Naumann et al , 2016).

Es requisito que el paciente tenga una anatomía normal de tórax y cuello, para la colocación de electrodos, y para realizar el cálculo se requiere de la velocidad de conducción de un estímulo eléctrico y la resistencia a la aplicación en el paciente. La principal desventeja consiste en que para el cálculo del gasto cardíaco se debe considerar valores de estabilidad hemodinámica, y la exactitud de dicho parámetro está influenciada por la presencia de fluidos en el tórax, la existencia de arritmias, la aparición de inestabilidad hemodinámica, las alteraciones en el hematocrito y los errores de la técnica en la detección de la señal de impedancia (Almela et al, 2015).

Hoy en día, el método de la la biorreactancia mejora al de bioimpedancia, ya que se basa en el análisis del cambio de fase que se produce en la onda eléctrica de alta frecuencia que es emitida al tórax y no en la medida de cambios de voltaje (Martín , 2016).

\section{Ecocardiografía y tecnología Doppler}

La tecnología consiste en la emisión de una onda con una frecuencia conocida a nivel cardíaco-vascular que choca con los eritrocitos en movimiento y se detecta por la presencia de una frecuencia diferente. Esta tecnología se basan en el cambio que se produce en la frecuencia de una onda acústica cuando el emisor y/o el receptor se encuentran en movimiento relativo, permitiendo obtener el GC de forma no invasiva y ofrece además una amplia información hemodinámica. A pesar de sus múltiples aplicaciones y la rápida extensión de su utilización en la UCl, la curva de aprendizaje aun es precoz, para poder garantizar la calidad y fiabilidad de las medidas (Martín , 2016).

\section{Doppler transtorácico}

En el año 2001 fue introducido en la práctica clínica, el sistema USCOM, el cual utiliza una sonda ciega sobre diferentes niveles del sistema cardiovascular (supraesternal, supraclavicular o paraesternal) en busca de los flujos sanguíneos máximos a nivel del tracto de salida de la válvula aórtica y pulmonar. Las áreas de los tractos se calculan a partir de un algoritmo antropométrico. En consecuencia, a partir de las velocidades y las áreas se pueden obtener las medidas de gasto cardíaco, volumen sistólico, índice cardíaco y resistencia vasculare (Martín, 2016).

\section{Doppler esofágico}

El sistema más utilizado el monitor CardioQ, aunque existen distintos dispositivos de doppler esofágico comercializados por varias empresas, con diferentes características técnicas según configuración y método de cálculo. Básicamente, el cálculo del gasto cardíaco con esta técnica, se utiliza un algoritmo que proyecta un flujo con proporción constante que abandona el corazón y continua bajando por la aorta torácica, aunque es una aproxim,ación no muy buena en pacientes conscientes.

\section{Ecocardiografía}

Esta tecnología consiste en ultrasonidos para evaluación de la morfología y cardiohemodinámica, la cual forma parte de la rutina diagnóstica en cardiología y por su

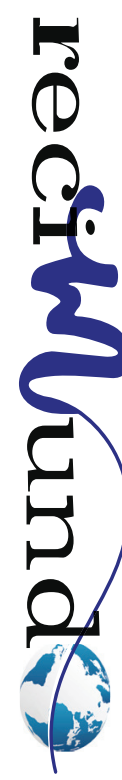


puesto, también implementada en UCl a pie de cama, evitando molestias a los pacientes y ofreciendo gran información a tiempo real. No obstante, no evalua la saturación venosa central y no permite estimar el tono vasomotor, y además la monitorización no puede ser continua (Martín, 2016).

\section{Técnica esCCO}

Es una técnica de monitorización hemodinámica realiza la determinación del gasto cardíaco de manera continua mediante los valores obtenidos de la señal de electrocardiograma (ECG) de cada ciclo, de la pulsioximetría, y de la onda de pulso periférico. Se fundamenta en que la onda de pulso, proporciona información relacionada con el tiempo (Tiempo de Tránsito de la Onda de Pulso PWTT), en la transmisión de la presión intravascular y en la información sobre el cambio de volumen de sangre arterial (NIHON KOHDEN, 2020).

\section{Monitoreo Hemodinámico Invasivo}

\section{Catéter de arteria pulmonar o de Swan- Ganz}

El catéter de la arteria pulmonar (CAP) o de Swan-Ganz es la técnica.que actualmente ha generado polémicas con respecto a su beneficio-costo en cuidados intensivos, aunque actualmente sigue considerándose la técnica estándar de referencia (gold standard) para la evaluación hemodinámica (Martín , 2016). Se fundamenta en la termodilución transcardíaca, según la ecuación de Stewart-Hamilton, inyectando un volumen de líquido (5 cc de suero salino) a temperatura inferior a la sanguínea, detectándose los cambios a lo largo del tiempo. El CAP proporciona datos clínicos como medición de gasto cardíaco (GC) y otras variables derivadas como la presión de arteria pulmonar (PAP), la presión de oclusión de arteria pulmonar (POAP) y parámetros de transporte (DO2) y consumo (VO2) de oxígeno de los tejidos (Martín, 2016).

\section{Análisis del contorno de pulso arterial}

Para que funcione esta técnica se requiere de la inserción de un catéter arterial (por lo general en una arteria periférica como la radial o en la arteria femoral) que se conecta a un transductor de presión. Se basa en el principio diferencial entre la presión sistólica y diastólica (presión de pulso) es proporcional al volumen sistólico e inversamente proporcional a la distensibilidad aórtica. A partir de allí, se pueden generar muchos algoritmos complejos fundamentados en varios modelos matemáticos diferentes, para generar el GC. De acuerdo a la periocidad de su aplicación deben calibrase ya sea manualmente la localización de la canulación arterial, los parámetros analizados y la exactitud con la que determinan el gasto cardíaco (Martín , 2016).

\section{Termodilución transpulmonar}

La técnica PiCCO (Pulse Induced Contour Cardiac Output) utiliza la termodilución transpulmonar y requiere de un catéter femoral que mide la presión arterial y los cambios de temperatura de la sangre, y de un catéter venoso central con un sensor capaz de medir la temperatura de la solución inyectada, cuyo volumen dependerá dependerá del peso corporal del paciente. Sefundamenta en la ecuación de StewartHamilton modificada para la obtención de parámetros, y para su calibración depende de la termodilución arterial o transpulmonar (Martín , 2016).

\section{Dilución de litio o litodilución transpulmo- nar}

Este sistema llamado LiDCO plus (Lithium Dilution Cardiac Output), permite medir el gasto cardíaco a partir de una onda de dilución, a través de la inyección de un bolo de cloruro de litio $(0,002-0,004 \mathrm{mmol} / \mathrm{kg})$ en un catéter venoso periférico o central. De esta manera, se detecta la concentración de litio en sangre arterial y el tiempo transcurrido desde su administración, calculando el gasto cardíaco, usando el área bajo la curva de concentración-tiempo. Se funadamenta en 
los principios de Stevart- Hamilton similares a la ecuación de la termodilución transpulnar (Martín , 2016).

\section{Sistema FloTrac ${ }^{\oplus} /$ Vigileo $^{\oplus}$}

Esta técnica utiliza el sensor FloTrac y el monitor Vigileo conectado a un catéter arterial radial o femoral, utilizando un algoritmo para proporcionar el gasto cardíaco continuo. Para ello emplea datos de presión arterial, la edad, el sexo y el área de la superficie corporal. Esta técnica ofrece los datos con una frecuencia de 20 segundos. El sensor calcula el volumen sistólico a partir de la presión de pulso. Cabe destacar que el algoritmo de FloTrac se ajusta continuamente, de acuerdo al tono vascular cambiante del paciente, no requiere una calibración manual (Martín , 2016).

\section{Sistema Most Care}

El sistema MostCare $\AA$ de Vygon (Vytech, Padova, Italia) utiliza el método Pressure Recording Analytical Method (PRAM), diferenciandose de otros algoritmos de análisis del contorno del pulso que calculan el área bajo la curva de la onda de pulso, tanto en la sístole como en la diástole, utilizando estos parámetros para la obtención de medidas de la impedancia sistémica, entre otros parámetros hemodinámicos. Se fundamenta en una versión modificada del algoritmo de Wesselings de análisis de la onda de pulso arterial. Se analiza la señal de pulso arterial antes de cada medición del gasto cardíaco, y no requiere calibración (Martín , 2016).

\section{Monitoreo Metabólico No Invasivo}

\section{Calorimetría Directa (CD)}

Entre las técnicas que nos permiten medir el gasto energético (GE) del ser humano está la calorimetría directa, la cual es considerada como el método más preciso para determinar el gasto energético en reposo(GER) (Arroyo, 2020). Consiste en colocar al paciente dentro de una cámara aislada y sellada, donde se cuantifica el calor generado por el individuo a través de los cam- bios de temperatura del aire y el agua que ingresan y salen de la cámara. Lamentablemente, es un método costoso, que requiere un equipo complejo y no es práctico, en el caso de pacientes en estado crítico, por lo que en la actualidad su principal uso es de tipo investigativo (Arroyo, 2020).

\section{Monitoreo Metabólico Invasivo}

\section{Calorimetría Indirecta (Cl)}

Usa la medición de la concentración de oxígeno y de dióxido de carbono para calcular el gasto energético usando la saturación de oxígeno arterial y la saturación de dióxido de carbono de la sangre venosa mixta, la cual se obtiene a través de un catéter en la arteria pulmonar, y aplicando de ecuaciones matemáticas (Arroyo, 2020).

\section{Control de Glucemia e Insulina}

A través de muestra de sangre, es menester un rutinario control de estos parámetros a haciendo uso de sistemas informáticos los cuales aplican de algoritmos para la monitorización de insulina. Actualmente, existen dispositivos que miden continuamente la glucemia mediante catéteres intravasculares (Arroyo, 2020).

\section{Monitoreo Neurológico No Invasivo}

\section{Evaluación Clínica}

Se buscan los parámetros que aporten una evaluación de estado y pronóstico como el diámetro pupilar, y la evaluación motora de la escala de coma de Glasgow, porque las alteraciones de estos parámetros se correlacionan directamente con el pronóstico de mortalidad, donde la alteraciones en diámetro y simetría pupilar el que guarda una relación más estrecha con ello (Carrillo E \& Garrido A, 2015).

\section{Monitorización mediante Doppler trans- craneal}

Se recomienda en todas las directrices del tratamiento moderno de la lesión cerebral traumática, para evaluar mejor, los umbra-

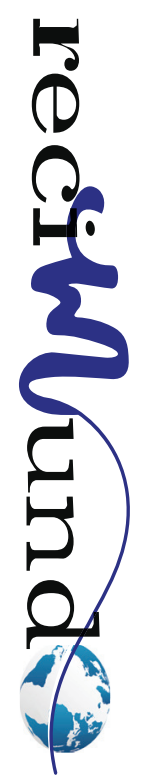


les de presión intracraneal que deben dar lugar a terapias específicas, así como la autorregulación cerebral (Carrillo E \& Garrido $A, 2015)$.

\section{Monitoreo Neurológico Invasivo}

\section{Monitorización de la presión intracraneal}

Vigila la presión dentro del cráneo a través de un dispositivo colocado dentro de la cabeza, cuyo valor debe mantenerse < de 20 $\mathrm{mmHg}$, y el cual es de suma importancia en algunos pacientes neurológicos, como por ejemplo los que han tenido una operación neuroquirúrgica complicada (Carrillo E \& Garrido A, 2015).

\section{Monitorización de la oxigenación cerebral y la saturación venosa yugular}

Actualmente, la tecnología permite la monitorización confiable y continua de la oxigenación regional cerebral, la cual debe encontrarse entre los valores normales promedio de 35 a $50 \mathrm{mmHg}$, ya que valores menores a $20 \mathrm{mmHg}$ nos indican isquemia cerebral. Por otro lado, medir la saturación venosa yugular cuyo valores normales oscilan entre 50-70\%., ofrecen información sobre el aporte y consumo de oxígeno de forma confiable, por lo que su monitorización continua es de suma importancia, para evitar episodios de desaturación que podrían pasar desapercibidos, y ocacionar daño daño (Carrillo E \& Garrido A, 2015).

\section{Conclusión}

El monitoreo del paciente crítico en la UCI es un control sistemático de variables fisiológicas que se miden con el objeto de detectar, reconocer y corregir tempranamente alteraciones de aparatos sistemas que podrían provocar posibles complicaciones, y puede consistir en técnicas invasivas como no invasivas.

Entre las técnicas de monitoreo respiratorio no invasivo se encuentran: el examen clínico a través de la observación, la oximetría de pulso y capnografía, las estimaciones de la mecánica del sistema respiratorio, la tomografía computarizada (TC) y la ecografía torácica. Mientras que entre las técnicas invasivas se encuentra la gasometría.

El monitoreo hemodinámico tiene técnicas no invasivas como: bioimpedancia-biorreactancia electrica torácica, la ecocardiografía y tecnología Doppler, el Doppler transtorácico, doppler esofágico, ecocardiografía y la técnica esCCO. Mientras que las técnicas invasivas son el catéter de arteria pulmonar o de Swan-Ganz, el Análisis del contorno de pulso arterial, la termodilución transpulmonar, la dilución de litio o litodilución transpulmonar, el sistema FloTrac y el sistema Most Care.

El monitoreo metabólico no invasivo, no es aplicable normalmente en $\mathrm{UCl}$ porque consite en la técnica de Calorimetría Directa (CD), la cual es sumamente impráctica hasta el momento. Pero dicho monitoreo cuenta con técnicas invasivas como la Calorimetría Indirecta $(\mathrm{Cl})$ y el control de glucemia e insulina.

Para el monitoreo neurológico no invasivo se aplican la evaluación clínica y la monitorización mediante Doppler transcraneal. Mientras que tambien son necesariamente aplicables técnicas invasivas como la monitorización de la presión intracraneal y la monitorización de la oxigenación cerebral y la saturación venosa yugular.

En definitiva, existen diferentes técnicas de monitorización invasivas o no invasivas disponibles, que cumplen con su propósito esencial dependiendo del conocimiento y experticia de los profesionales intensivista que la utilicen, así como también de los recursos tecnológicos con los que cuenta cada UCl. 


\section{Bibliografía}

Aguilar, C., \& Martinez, C. (2017). La Realidad de la Unidad de Cuidados Intensivos. Medicina Crítica, 31, 171-173.

Almela, A., Millán, J., Alonso, J., \& García, P. (2015). Monitorización hemodinámica no invasiva o mínimamente invasiva en el paciente crítico en los servicios de urgencias y emergencias. Emergencia, $1-27$

Arroyo, A. (2020). Calorimetría Indirecta en Cuidados Críticos: una revisión narrativa. Revista de Nutrición Clínica y Metabolismo, 1-27.

CareTaker. (2020). Care Taker Medical. Recuperado el 19 de 08 de 2021, de https://caretakermedical. net/es/sobre-nosotros-caretaker-medical/

Carrillo E, \& Garrido A. (2015). Monitoreo neurológico multimodal. Rev Mex de Anestesiología, 38(3), 425-426.

Cediel, X., Rebellón, D., \& Yaset , C. (2020). Enfoque del paciente crítico y ventilación mecánica para no expertos. Boyacá: Búhos Editores Ltda.

Citerio G, Oddo M, \& Taccone FS. (2015). Recommendations for the use of multimodal monitoring in the neurointensive care unit. Curr Opin Crit Care, 21, 113-119.

Crashing Patient. (03 de 04 de 2020). Emergency Medicine and ED Critical Care. Obtenido de https://crashingpatient.com/procedures/central-line-placement.htm/

De Waele , E., Honoré , P., \& Malbrain , M. (2018). Does the use of indirect calorimetry change outcome in the ICU? Yes it does. Curr Opin Clin Nutr Metab Care, 21(2), 126-129.

Jones JG, \& Jones SE. (2020). Discriminating between the effect of shunt and reduced VA/Q on arterial oxygen saturation is particularly useful in clinical practice. J Clin Monit Comput, 37, 337-350.

Kipnis, E., Ramsingh, D., \& Bhargava, M. (2015). Critical Care Monitoring. Critical Care Research and Practice, 21-29.

Maqueda, M., \& Pérez, E. (2018). Volumen de sangre extraído al paciente crítico las primeras $24 \mathrm{~h}$ de ingreso. Enfermería Intensiva, 29(1), 14-20.

Márquez, Pámanes, González, Márquez, Gómez, Negrete, \& Muñoz. (2012). Lo que debe conocerse de la gasometría durante la guardia. Rev Med Inst Mex Seguro Soc, 50 , 389-396.
Martín , E. (2016). Técnicas para la monitorización hemodinámica en las Unidades de Cuidados Intensivos. Técnicas no invasivas. Andalucía: Informes de Evaluación de Tecnologías Sanitarias.

Motta, L., Barrera, M., Peña, C., \& Tamaríz, O. (2017). Monitorización de oxigenación tisular. Conferencias Magistrales, 40(1), 350-364.

Naumann, D., Midwinter, M., Midwinter, M., \& Hutchings, S. (2016). Venous-to-arterial CO2 differences and the quest for bedside point-of-care monitoring to assess the microcirculation during shock. Ann Transl Med 2016, 4(2), 37.

NIHON KOHDEN. (2020). Tecnologías Innovadoras. Recuperado el 19 de 08 de 2021, de https://eu.nihonkohden.com/es/innovativetechnologies/escco/ whatisescco.html

Ortiz , G., \& Dueñas, R. (2019). Anatomía y monitorización respiratoria. Argentina: Editorial Medica Panaméricana.

Robba, C., Graziano, F., \& Rebora, P. (2021). Intracranial pressure monitoring in patients with acute brain injury in the intensive care unit (SYNAPSE-ICU): an international, prospective observational cohort study. The Lancet Neurology, 20(7), 548-558.

Sessler, D. I. (2008). Temperature monitoring and perioperative thermoregulation. Anesthesiology, 109(2), 318-338.

Singer, P., Blaser, A., Berger, M., \& Alhazzani, W. (2019). ESPEN guideline on clinical nutrition in the intensive care unit. Clin Nutr, 38(1), 48-79.

Warttig, S., Alderson, P., Evans, D., \& Lewis, S. (2018). Automated monitoring compared to standard care for the early detection of sepsis in critically ill patients. Cochrane Library.

Weiner, R., Ryan, E., \& Yohannes, J. (2021). Critical Care. McGraw Hill. 
MORENO SASIG, N. G., VÉLEZ MUENTES, J. R., CAMPUZANO FRANCO, M. A., ZAMBRANO CÓRDOVA, J. R., \& VERA PINARGOTE, R. G.

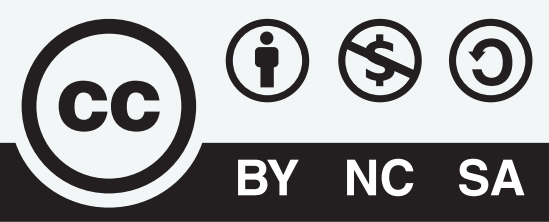

CREATIVE COMMONS RECONOCIMIENTO-NOCOMERCIAL-COMPARTIRIGUAL 4.0.

\section{CITAR ESTE ARTICULO:}

Moreno Sasig, N. G., Vélez Muentes, J. R., Campuzano Franco, M. A., Zambrano Córdova, J. R., \& Vera Pinargote, R. G. (2021). Monitorización invasiva y no invasiva en pacientes ingresados a UCl. RECIMUNDO, 5(3), 278-292. https://doi.org/10.26820/recimundo/5.(2).julio.2021.278-292 